\title{
Improving the classification of urban tree diversity from Very High Spatial Resolution hyperspectral images: comparison of multiples techniques
}

\author{
BRABANT Charlotte \\ LETG-Rennes UMR 6554 CNRS \\ University of Rennes \\ Rennes , France \\ charlotte.brabant@yahoo.fr
}

\author{
ALVAREZ-VANHARD Emilien \\ LETG-Rennes UMR 6554 CNRS \\ University of Rennes \\ Rennes, France \\ emilien.alvarez@univ-rennes2.fr
}

\author{
HOUET Thomas \\ LETG-Rennes UMR 6554 CNRS \\ University of Rennes \\ Rennes, France \\ thomas.houet@univ-rennes2.fr
}

\begin{abstract}
The aim of the study is to compare and assess the efficiency of conventional hyperspectral techniques (dimension reduction, learning and classification methods) to classify the urban tree vegetation diversity. A specific focus is made using very high spatial resolution hyperspectral images acquired from airborne sensor and simulated at various spatial/spectral resolutions. Results show that $\mathbf{7 8 . 8 \%}$ of overall accuracy can be reached using MNF and SVM methods to classify urban tree diversity. Moreover, results are more sensitive to learning methods rather than dimension reduction or classification ones. Finally, VHRS hyperspectral imagery are promising for such a key urban environmental issue.
\end{abstract}

Keywords - Tree species, urban area, reduction dimension, classification, learning methods.

\section{INTRODUCTION}

In the context of urban sprawling and increasing and population, urban vegetation has several interests: it provides many services such as reducing the urban heat island (UHI) through shade, evapotranspiration and tree photosynthesis [1, 2]. It improves air quality or even habitat biodiversity [3]. Urban vegetation also has disservices such as allergies due to pollen, constitutes a refuge for harmful bird species for inhabitants (noise, droppings, etc...). The intensity of these services and disservices depends on the plant species, its location and structure [4-6]. For example, the effectiveness of UHI mitigation depends on the water needs of the species concerned [7].

Mapping tree species therefore responds to major urban environmental issues. Remote sensing applied in urban areas makes it possible to overcome the lack of reliable and reproducible information on vegetation to evaluate more precisely ecosystem services $[8,9]$. The existing literature has showed the potential of Very High Spatial Resolution (VHSR) multispectral data to discriminate trees species [1012], as well as VHRS hyperspectral imageries [13-16] associated with Lidar or not. However, the high dimensionality of VHRS hyperspectral data can alter the effectiveness of conventional methods to discriminate against urban tree diversity.

The French Space Agency is currently considering developing a new spaceborne VHRS hyperspectral sensor: HYPXIM. The objective of this study is to evaluate (1) the ability of VHSR hyperspectral data to classify urban tree diversity and (2) the most effective methods.

\section{DATA}

A. Study area

The study site is the city center of Toulouse (4336'16' N, 1'26'39' 'E) - France. The urban area is highly artificialized and the vegetation is embedded between buildings and houses, present along roads or in courtyards.

\section{B. Data}

Airborne hyperspectral data were acquired using the HySpex sensor ( $2 \mathrm{~m}$ spatial resolution, $>480$ spectral bands ranging from 0.4 to $2.5 \mu \mathrm{m}$ ) in July 2015 . In order to assess the potential of the HYPXIM spaceborne sensor (192 bands), HySpex data have been resampled accordingly to HYPXIM specifications at $4 \mathrm{~m}$ and $8 \mathrm{~m}$ spatial resolution. A Sentinel 2 imagery at $10 \mathrm{~m}$ resolution has also been simulated for the sake of the demonstration. Atmospheric corrections were applied using the Cochise method.

A field campaign has been conducted between $31^{\text {st }}$ may and $2^{\text {nd }}$ June, 2017. More than 600 trees have been inventoried and geolocated, using a differential GPS (with an absolute geometric accuracy below $10 \mathrm{~cm}$ ). It is assumed that the tree composition did not changed between the airborne acquisition (2015) and the field survey (2017). In addition, the dataset is very unbalanced: for e.g. Platanus $x$ hispanica have 144 individuals unlike other species like Prunus $x$ avium which have only 7 individuals. Although this unbalanced dataset is representative of the data generally found in urban areas, it can distinguish 12 to 19 families, and from 14 to 27 species respectively, while considering a minimum number of 4 and 10 individuals for each of them.

\section{METHODS}

Figure 1 summarizes the overall approach to assessing the influence of reduction dimension, learning and classification methods. Their influence is evaluated on the classification performance for two levels of nomenclature (Species and Families), themselves subdivided into 2 sublevels depending on the minimum number of individuals per classes to be considered (respectively 4 and 10).

\section{A. Reduction dimention methods}




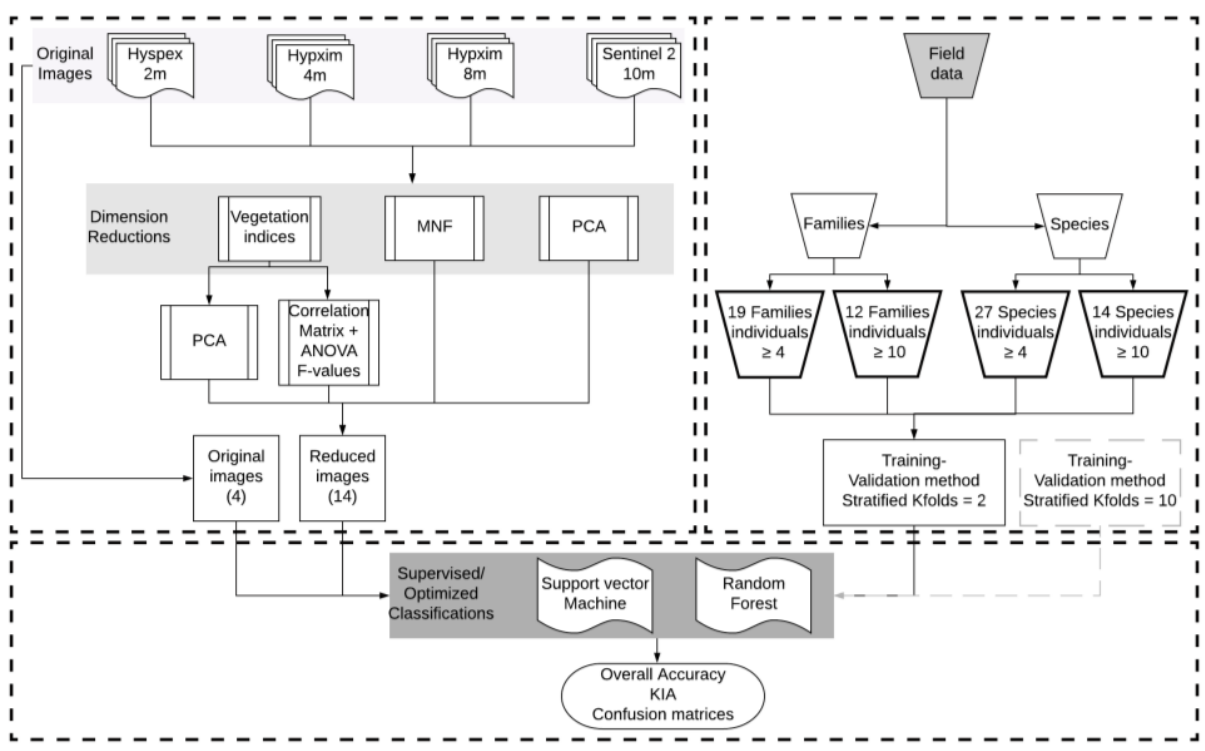

Fig. 1. General methodological approach

Several dimension reduction methods have been applied on the four considered dataset: PCA (Principal Component Analysis), MNF (Minimum Noise Fraction) and an innovative method to select the most discriminant meaningful vegetation indices. 96 spectral vegetation indices (VIs) inventoried by [17] were computed for the three hyperspectral datasets only. It is assumed that these VIs characterizing the biogeochemical properties of plants can contribute to better discriminate trees. The selection of VI was made using two methods: (1) PCA method is used to eliminate the correlation between VIs. Kaiser's criterion [18] is used for the selection and components with a eigenvalue greater than 1 are selected; (2) The K-best method uses the F-value of ANOVA (ANalysis Of VAriance) as a ranking criterion. From a correlation matrix (Pearson's r), if the correlation between two VIs is greater than $85 \%(r>0,85)$, the one with the smallest F-value is removed from the selection.

For the sake of the demonstration, corresponding results are provided only for the sub-levels of the 19 and 12 families and compared using the Overall Accuracy (OA) and the Kappa Index of Agreement (KIA).

\section{B. Training data and methods}

Each classification is trained and validated using with stratified Kfolds method applied thanks to the in situ dataset. For each individual, vectors were extracted from reduced and unreduced images. It is randomly partitioned into $\mathrm{k}$ subsamples with the same proportion of individuals per class. The first method uses Kfolds $=2$ (2-folds): the dataset is separated into $50 \%$ training and $50 \%$ validation samples. The second method defines Kfolds $=10$ (10-folds), meaning that $10 \%$ is used for the validation and $90 \%$ for the training, iteratively. This 10-folds methods is applied only for the sublevel with minimum individuals $=10$. Each of these two methods is applied 10 times for each dataset.

For the sake of the demonstration, the 2-folds method is used as a training method on all reduced and unreduced image datasets while the 10-folds is applied only on the one exhibiting the best $\mathrm{OA}$ with the 2-folds and for the sub-level of the 14 species (i.e. with at least 10 individuals).

\section{Influence of classification methods}

Two commonly used classifiers were tested and compared: SVM (Support Vector Machine) and RF (Random Forest). SVM classifier is adopted here as it might be sensitive to small and non-homogeneous learning datasets compared to RF for instance [9]. SVM parameters (kernel functions, constant $\mathrm{C}$, etc.) have been optimized because they can affect the quality of the training [19]. The RF classifier is also known to be a good classifier for hyperspectral data [20] because it tends to select or weight characteristics during learning [21]. Three parameters of RF have been optimized here to increase the predictive power of the model: the number of tree forests, the number of maximum features, and the criterion.

\section{RESULTS}

\section{A. Influence of reduction dimension and classification methods}

The number of selected components from MNF and PCA is respectively of 40 and 30 for all hyperspectral images and of 9 and 13 components for Sentinel 2, representing at least $98 \%$ of the variance. Using the PCA on VIs provides a selection of 19 vegetation index for HySpex $2 \mathrm{~m}$ (explaining $86.16 \%$ of the variance), 19 for HYPXIM 4m (84.5\%) and 17 for HYPXIM $8 \mathrm{~m}(85.89 \%)$. When using the ANOVA and the correlation matrix, 45 uncorrelated VIs are selected for HySpex 2m, 39 for HYPXIM 4m and 38 for HYPXIM 8m. Indices with the highest value regardless of the resolution mainly rely to the Chlorophyll content with 24/45 selected indices for HySpex, 19/39 for HYPXIM 4m and 24/38 for HYPXIM 8m

Reduced datasets using MNF provide best results no matter the nomenclature, the number of individuals, classifiers or datasets used (Table 1). For the "12 Families" sub-level (i.e. individuals $>10$ ), the mean OA is greater than $60 \%$. VI selection have close results with $\mathrm{OA}=58.17 \%$ $(\mathrm{KIA}=0.50)$ for the HySpex image and RF. PCA provides worst results than any other dimension reduction methods. Reduced datasets provide always better results than nonreduced ones, except for Sentinel 2 image in some cases. Both SVM and RF classifiers offer similar results. Even if RF gives generally the best OA regardless of the nomenclature, the highest OA are obtained with SVM with at 
least 10 individuals per class, ranging from $59 \%$ to $65 \%$ with MNF reduced images.

TABLE I. CLASSIFICATION RESULTS USING SVM AND RANDOM FOREST ON THE TWO SUB-LEVELS OF FAMILY TREES DIVERSITY, USING VARIOUS UNREDUCED AND REDUCED HYPERSPECTRAL DATASETS AND THE 2-FOLDS LEARNING METHOD.

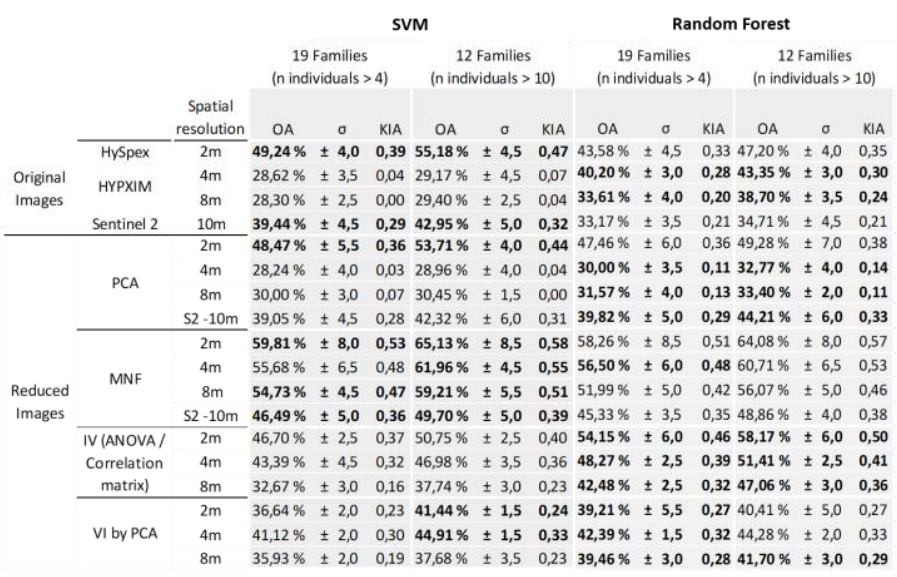

\section{B. Influence of learning methods}

The 10-folds learning method applied on MNF image datasets strongly improves OA: $+15 \%$ for the HySpex $2 \mathrm{~m}$, $+14 \%$ for HYPXIM $4 \mathrm{~m},+4 \%$ for HYPXIM $8 \mathrm{~m}$ and $+9 \%$ for Sentinel 2 compared with the 2-folds method (Figure 2). Similar results are obtained with HySpex 2m and HYPXIM $4 \mathrm{~m}$ with the SVM classifier $(\mathrm{OA}=78.38 \%, \mathrm{KIA}=0.73)$. Classification accuracies are similar with RF: $75.68 \%$ with HySpex $2 \mathrm{~m}$ and $72.97 \%$ with HYPXIM $4 \mathrm{~m}$. Those for HYPXIM 8m and Sentinel2 datasets do not exceed $65 \%$ (OA $=64.86 \%$ and $62.16 \%$ respectively with RF).

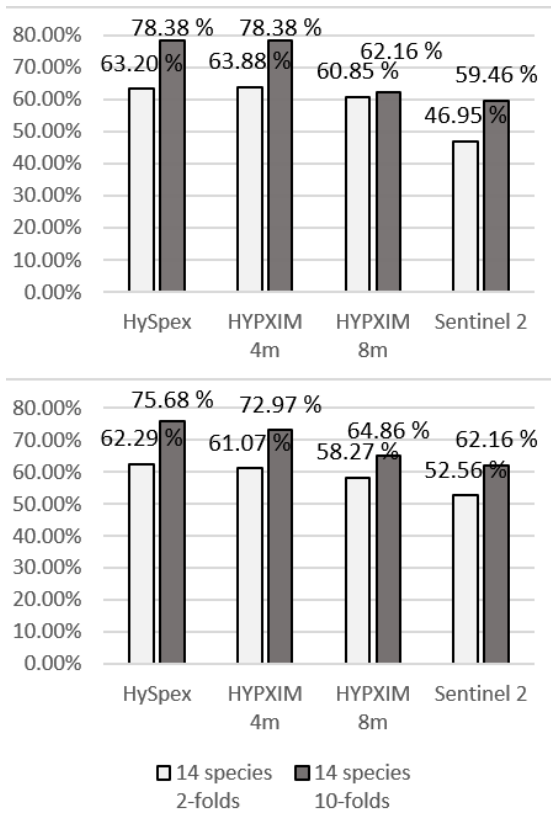

Fig. 2. Results of SVM (top) and RF (bottom) classifiers applied on MNF reduced datasets with 2 -Folds and 10-Folds learning methods with the 14 Species nomenclature.

\section{DISCUSSION}

This study confirms the literature on the influence of the training data and methods. Interclass and intraclass variability inheriting from an unbalance dataset can have strong effects. Although it is quite common in urban area to observe few dominating species due to urban planning and management strategies and individual choices of inhabitants, a greater number of folds prevent from over-fitting that may inherit from unbalanced urban training datasets. Conversely, depending on the considered objectives, the choice of hyperspectral techniques can be adapted. For instance, we could recommend the use of SVM classifier, applied on MNF reduced image data with a limited number of samples per class if the objective is to map a dominant allergenic tree species. In any case, this study highlights that training samples have to be clearly designed and optimized prior to hyperspectral processing.

Another interesting result comes from the evaluation of the respective influence of hyperspectral methods for improving the classification of tree diversity in cities. Training methods have a greater influence than reduction dimension and classification methods.

This study highlights that VHRS hyperspectral imageries with a spatial resolution of 4 or 8 meters can be very effective for urban tree diversity classification. First of all, it equals or overcomes OA rates obtained in others studies for AVIRIS, AISA or MIVIS hyperspectral sensors exhibiting a finer spatial resolution with a similar / greater number of tree families [13-16]. While one can expect an improvement of tree classification with an increasing spatial resolution, results suggest that HYPXIM (4m) can be as appropriate as HySpex $(2 \mathrm{~m})$ imageries. A finer resolution may involve a greater spectral intra-class variability in tree crowns that can lead to misclassifications [21]. Indeed, spectral reflectance may vary within a single tree, depending on biochemistry and water content [22, 23], solar exposure of leaves, and shaded effects of street orientation or neighbouring building height. Figure 3 shows that at 2 meters resolution, shadows from building or even from London plane alignments are misclassified as Linden species, which is the second dominant tree species in Toulouse.

Finally, obtained results are promising, although there is still room for improvement. Indeed, July may not have been an optimal period for hyperspectral data acquisition and urban tree species differentiation. Spaceborne hyperspectral time series of hyperspectral imageries would undoubtly contribute to a huge step forward into urban tree species classification.

\section{CONCLUSION}

The objective of this study was to evaluate the ability of VHSR hyperspectral data to discriminate between urban tree species and/or families and the related most effective methods. It shows that (1) dimensional reduction methods are essential, (2) learning methods contribute significantly to improving the classification of urban tree species and even more (3) the type of classifiers - SVM and RF - does not have a significant influence. It stressed the importance of the in situ dataset which requires particular attention due to the high diversity of tree species in urban areas and with regard to the expected objectives. A spaceborne hyperspectral VHRS (4 meters) sensor would be particularly promising for urban environmental issues as times series would contribute to a huge step forward. 


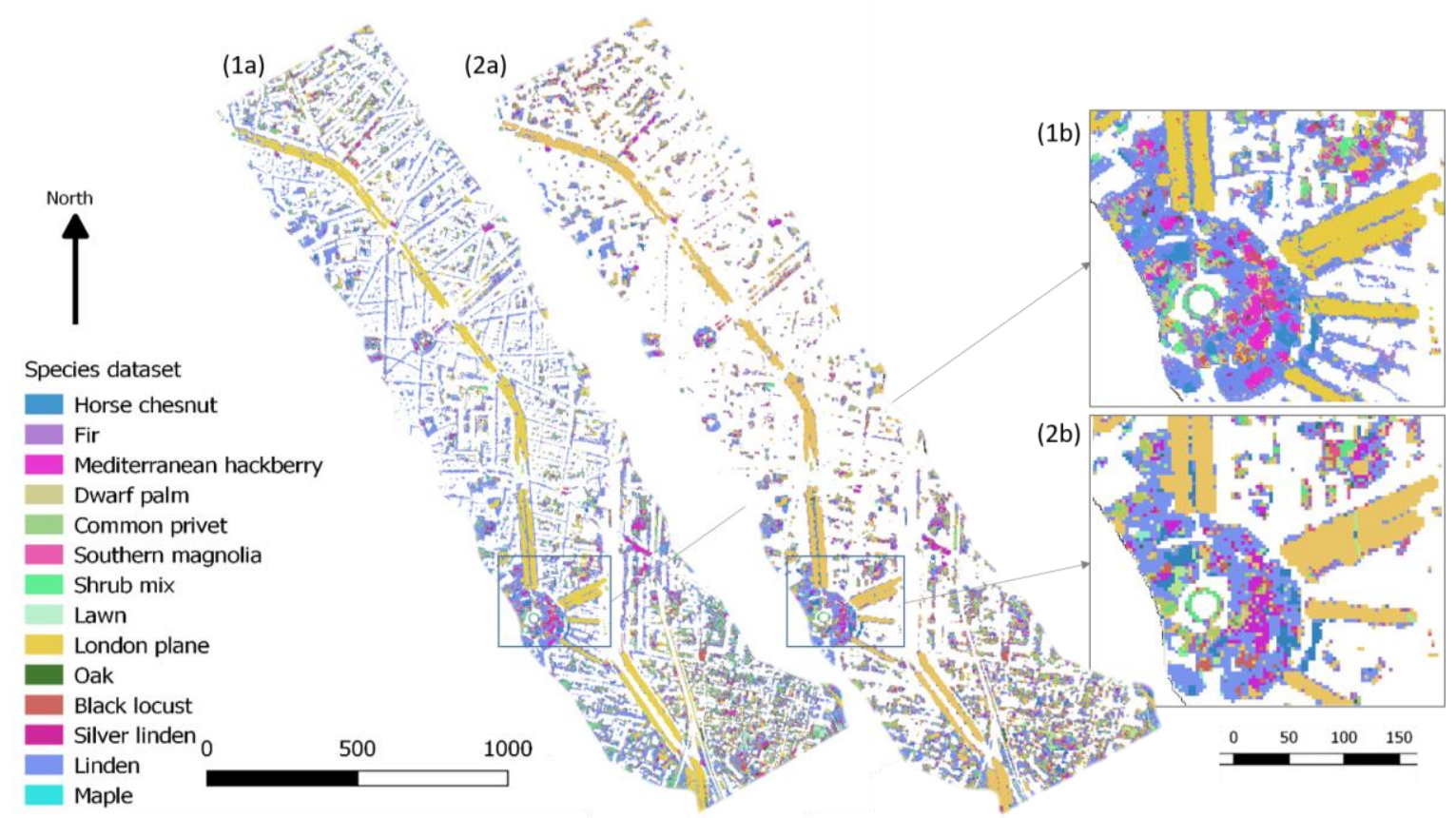

Fig. 3. Results of SVM classifications applied with MNF reduced datasets with 10-Folds method with (a) HySpex 2 meters resolution imageres and (b) HYPXIM 4 meter resolution, on (1) the entire study area and with (2) a zoom-in on the "Grand-Rond" urban park exhibiting one of the greatest urban tree diversity.

\section{ACKNOWLEDGMENT}

This study has been founded by the National Research Agency of France - ANR HYEP 'Hyperspectral imagerY for Environmental urban Planning” ANR 14-CE22-0016-01.

\section{REFERENCES}

[1] Akbari, H.; Pomerantz, M.; Taha, H. Cool surfaces and shade trees to reduce energy use and improve air quality in urban areas. Solar Energy 2001, 70, 295-310.

[2] Bolund, P.; Hunhammar, S. Ecosystem services in urban areas. Ecological economics 1999, 29, 293-301.

[3] Santamouris, M. Using cool pavements as a mitigation strategy to fight urban heat island-A review of the actual developments. Renewable and Sustainable Energy Reviews 2013, 26, 224-240, doi:10.1016/j.rser.2013.05.047.

[4] Escobedo, F. J.; Nowak, D. J. Spatial heterogeneity and air pollution removal by an urban forest. Landscape and Urban Planning 2009, 90, $102-110$.

[5] McCarthy, H. R.; Pataki, D. E. Drivers of variability in water use of native and non-native urban trees in the greater Los Angeles area. Urban Ecosyst 2010, 13.

[6] McPherson, E. G.; Simpson, J. R.; Xiao, Q.; Wu, C. Million trees Los Angeles canopy cover and benefit assessment. Landscape and Urban Planning 2011, 99, 40-50.

[7] Lemonsu, A.; Masson, V.; Shashua-Bar, L.; Erell, E.; Pearlmutter, D. Inclusion of vegetation in the Town Energy Balance model for modelling urban green areas. Geoscientific Model Development 2012, 5, 1377-1393.

[8] Davies, Z. G.; Edmondson, J. L.; Heinemeyer, A.; Leake, J. R.; Gaston, K. J. Mapping an urban ecosystem service: quantifying above-ground carbon storage at a city-wide scale. Journal of Applied Ecology 2011, 48, 11251134.

[9] Weng, Q., Quattrochi, D. A.; Carlson, T. Remote sensing of urban environments: Special issue. Remote Sensing of Environment 2012, 117, $1-2$.

[10] Immitzer, M.; Atzberger, C.; Koukal, T. Tree Species Classification with Random Forest Using Very High Spatial Resolution 8-Band WorldView-2 Satellite Data. Remote Sensing 2012, 4, 2661-2693.

[11] Key, T. A Comparison of Multispectral and Multitemporal Information in High Spatial Resolution Imagery for Classification of Individual Tree Species in a Temperate Hardwood Forest. Remote Sensing of Environment 2001, 75, 100-112.

[12] Pu R., Landry S. A comparative analysis of high spatial resolution IKONOS and WorldView-2 imagery for mapping urban tree species. Remote Sensing of Environment 2012, 124, 516-533.

[13] Alonzo, M.; Bookhagen, B.; Roberts, D.A. Urban tree species mapping using hyperspectral and lidar data fusion. Remote Sensing of Environment 2014, 148, 70-83.

[14] Voss M., Sugumaran, R. Seasonal Effect on Tree Species Classification in an Urban Environment Using Hyperspectral Data, LiDAR, and an ObjectOriented Approach. Sensors 2008, 8, 3020-3036.

[15] Xiao, Q.; Ustin, S. L.; McPherson, E. G. Using AVIRIS data and multiplemasking techniques to map urban forest tree species. International Journal of Remote Sensing 2004, 25, 5637-5654.

[16] Boschetti, M.; Boschetti, L.; Oliveri, S.; Casati, L.; Canova, I. Tree species mapping with Airborne hyper-spectral MIVIS data: the Ticino Park study case. International Journal of Remote Sensing 2007, 28, 1251-1261.

[17] Erudel, T.; Fabre, S.; Houet, T.; Mazier, F.; Briottet, X. Criteria Comparison for Classifying Peatland Vegetation Types Using In Situ Hyperspectral Measurements. Remote Sensing 2017, 9, 748.

[18] Kaiser, H.F. The Application of Electronic Computers to Factor Analysis. Educational and Psychological Measurement 1960, 20, 141-151.

[19] Camps-Valls, G.; Bruzzone, L. Kernel Methods for Remote Sensing Data Analysis. 2009, 15.

[20] Ham, J.; Yangchi Chen; Crawford, M. M.; Ghosh, J. Investigation of the random forest framework for classification of hyperspectral data. IEEE Transactions on Geoscience and Remote Sensing 2005, 43, 492-501, doi:10.1109/TGRS.2004.842481.

[21] Dalponte, M.; Orka, H. O.; Gobakken, T.; Gianelle, D.; Naesset, E. Tree Species Classification in Boreal Forests With Hyperspectral Data. IEEE Transactions on Geoscience and Remote Sensing 2013, 51, 2632-2645.

[22] Cochrane, M. A. Using vegetation reflectance variability for species level classification of hyperspectral data. International Journal of Remote Sensing 2000, 21, 2075-2087.

[23] Ustin, S. L.; Gitelson, A. A.; Jacquemoud, S.; Schaepman, M.; Asner, G. P.; Gamon, J. A.; Zarco-Tejada, P. Retrieval of foliar information about plant pigment systems from high resolution spectroscopy. Remote Sensing of Environment 2009, 113, S67-S77. 